NASA TECHNICAL NOTE

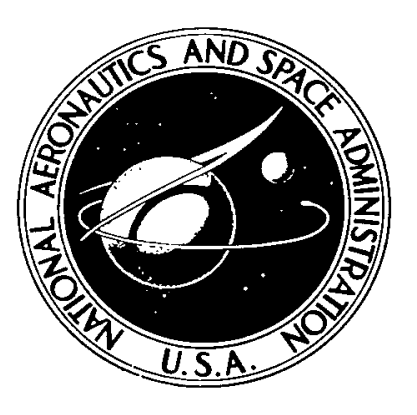

NASA IN D-7036

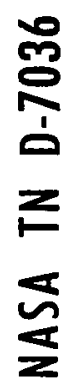
C.I

LOAN COPY: RETURP

AFWL (DOGL)

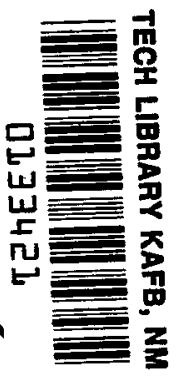
KIRTLAinD AFB, N. T..

\title{
ON A TRANSFORMATION OF THE DIFFERENTIAL EQUATIONS OF THE LUNAR THEORY
}

by Peter Musen

Goddard Space Flight Center Greenbelt, Md. 20771 


\begin{abstract}
1. Report No.
TASA TN D-7036

4. Title and Subtitle

On a Transformation of the Differential Equations of the Lunar Theory
\end{abstract}

7. Author(s)

\section{Peter Musen}

9. Performing Organization Name and Address

Goddard Space Flight Center

Greenbelt, Maryland 20771

12. Sponsoring Agency Name and Address

National Aeronautics and Space Administration

Washington, D. C. 20546

15. Supplementary Notes
3. Recipient's Catalog No.

5. Report Date

February 1971

6. Performing Organization Code

8. Performing Organization Report No. G-1007

10. Work Unit No.

11. Contract or Grant No.

13. Type of Report and Period Covered Technical Note

14. Sponsoring Agency Code

16. Abstract

The Hill-Brown differential equations of satellite coordinates are transformed to a type which can be integrated using an analytic programming language. The differential equation for the parallax of a satellite is also established. Its use facilitates computation of Hill's periodic intermediary orbit and provides a good check for the expansion of the coordinates and frequencies. Knowledge of the expansion of the parallax facilitates the formation of differential equations for terms with a given characteristic. These differential equations are put into a form solvable by iteration on the computer. As in the classical theory we obtain expansions of the coordinates and parallax in the form of trigonometric series in four arguments and in powers of the constants of integeration. We expand the differential operators into series in squares of the constants of integration. Only the zero order terms in these expansions are used to integrate the differential equations. The remaining terms produce the crosseffects between perturbations of different order. We apply the averaging operator to the right sides of the differential equations to deduce the expansion of the frequencies in powers of squares of the constants of integration.

17. Key Words Suggested by Author

Hill-Brown differential equations

Satellite coordinates expansion

Satellite parallax expansion
19. Security Classif. (of this report)

Unclassified
20. Security Classif. (of this page)

Unclassified
18. Distribution Statement Unclassified-Unlimited

\footnotetext{
*For sale by the National Technical Information Service, Springfield, Virginia 22151
} 


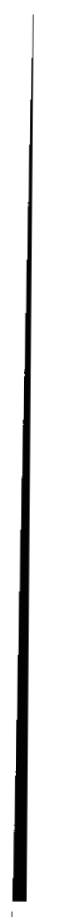




\section{CONTENTS}

Basic Notation. ................

INTRODUCTION $\ldots \ldots \ldots \ldots \ldots \ldots \ldots \ldots \ldots$

THE BASIC DIFFERENTIAL EQUATIONS....... 3

VARIATIONAL SOLUTION. . . . . . . . . . . 14

THE TERMS DEPENDING UPON THE FIRST

POWER OF THE ECCENTRICITY . . . . . . . . 16

HIGHER ORDER TERMS IN $\xi$ AND $\eta \ldots \ldots \ldots 20$

PERTURBATIONS IN THE $z$-COORDINATE $\ldots \ldots .25$

CONCLUSION $\ldots \ldots \ldots \ldots \ldots \ldots \ldots \ldots \ldots \ldots \ldots$

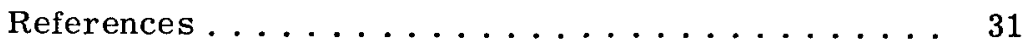




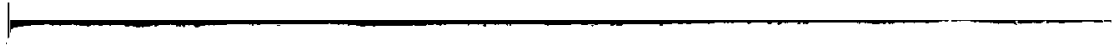




\section{BASIC NOTATION}

f - the gravitational constant,

$\mathrm{E}$ - the mass of the planet,

M - the mass of the satellite,

t - dynamical time,

$\mathrm{x}, \mathrm{y}, \mathrm{z}$ - planetocentric coordinates of the satellite,

$u=x+y \sqrt{-1}$,

$\mathrm{s}=\mathrm{x}-\mathrm{y} \sqrt{-1}$

$\rho$ - the planetocentric distance of the satellite,

$\mathbf{w}=1 / c$,

$\rho_{0}$ - the variational part of $\rho$,

$w_{0}$ - the variational part of $w$,

$\mathrm{n}$ - the mean daily sidereal motion of the satellite,

a - the mean semi-major axis of the satellite defined by means of the Kepler relation

$$
a^{3} n^{2}=f(E+M),
$$

a - the mean semi-major axis defined as the constant factor attached to the variational solution,

e - the constant of the eccentricity of the satellite,

$\gamma$ - the sine of one half of the orbital inclination of the satellite relative to the orbit of the Sun,

$c\left(n-n^{\prime}\right)$ - the anomalistic frequency of the satellite,

$c_{0}$ - the part of $c$ independent from e, e', $y$ and $a$,

$g\left(n-n^{\prime}\right)$ - the draconitic frequency of the satellite,

$\mathrm{g}_{0}$ - the part of $\mathrm{g}$ independent from e, e', $\gamma$ and $\alpha$,

$\zeta=\exp \left(n-n^{\prime}\right) t \sqrt{-1}$,

$D=\zeta d / d \zeta$,

$e^{\prime} \quad$ - the eccentricity of the solar planetocentric orbit, 
$a^{\prime} \quad$ - the semi-major axis of the solar orbit,

$n^{\prime} \quad$ - the mean daily motion of the sun in its orbit around the planet,

$m=n^{\prime} /\left(n-n^{\prime}\right)$,

$a=a / a^{\prime}-$ the parallactic factor,

$\Omega \quad$ - the disturbing function. 


\title{
ON A TRANSFORMATION OF THE DIFFERENTIAL EQUATIONS OF THE LUNAR THEORY
}

\author{
by \\ Peter Musen \\ Goddard Space Flight Center
}

\section{INTRODUCTION}

In recent times electronic machines have been extensively applied to develop analytical theories of satellites. The works by Barton (1966) and Broucke (1968) on the analytical expansion of the lunar disturbing function, by Broucke and Garthwaite (1969), Charpont and Mangency (1965), Danby et al (1965) on the manipulation of the literal series on the machine, by Deprit (1969) and Kamel (1969) on the application of Lie series to the elimination of the short period terms from a Hamiltonian, a beautiful work by Deprit and Henrard on the duplication of the Delaunay theory (1970), and also the work by Elmabsout (1970) on a semi-numerical method in the lunar theory deserve special attention.

In this article, we suggest a transformation of the differential equations of the Hill $(1878,1886)$ and Brown (1896-1899) theory of satellites to a form integrable by the process of iteration. We retain the classical form of expansion of rectangular coordinates into trigonometric series in four arguments and powers of the satellite's mean orbital eccentricity e, sine of one half of the mean orbital inclination $\gamma$, eccentricity of the solar orbit $e^{\prime}$, and parallactic factor $\alpha=a / a^{\prime}$. The planetocentric orbit of the sun is assumed to be elliptic. We differ from the classical approach in the form of the differential equations and method of integration.

Hill and Brown use the method of undetermined coefficients to integrate the differential equation for terms with a given characteristic. They form a set of equations satisfied by the coefficients of the expansion of coordinates, and solve these equations by iteration. This way of determining the coefficients is especially convenient if the computation is performed by using a desk calculator. At present, the explicit formulation of these equations in detail cannot be considered necessary.

We now have analytic programming languages which permit the process of iteration to be applied directly to the differential equations. To make this application possible, we transform Hill's differential equations for the complex coordinates to a form containing on the left-hand side a linear differential operator with constant coefficients, and on the right-hand side the small parameter $\mathrm{m}^{2}$ as a factor. Such a form allows the application of the method of iteration and guarantees its fast convergence with the accepted numerical accuracy.

Besides the differential equations for the coordinates, we also establish (Andoyer, 1926) the differential equation for the parallax. This equation is redundant, but its use provides a good check of our results. In particular, we found that by the combined use of the differential equations for the coordinates and for parallax, we can determine the periodic variational solutions by the method of iteration, thus by-passing Hill's equations for the coefficients of the variational terms. The same set of equations can be used to determine the terms containing the first power of the eccentricity 
as a factor, providing we know the main part $c_{0}$ of the anomalistic frequency $c$. We can derive $c_{0}$ by supression of certain terms in the expansion of the parallax.

We suggest the use of the expansion of Andoyer (1926) for the main parts of the anomalistic and draconitic frequencies. Using the iterative procedure for the terms of higher order, we determine, at each iteration step, the coefficients of terms with a given characteristic from the set of pairs of linear equations with two unknowns. These equations are automatically formed and solved by the machine.

We derive the higher order terms in the expansion of $\mathrm{c}$ and $\mathrm{g}$ in powers of $\mathrm{e}^{2}, \mathrm{e}^{2}, \gamma^{2}$ and $a^{2}$ by applying the averaging process to the equations of motion written in a suitable form. A close look at the integration procedure of the Hill-Brown theory shows that integration consists of applying the inverses of some quadratic differential operators with constant coefficients to the right-hand sides of the differential equations of motion. These operators represent the first terms of the expansions in powers of $\mathrm{e}^{2}, \mathrm{e}^{12}, \gamma^{2}$ and $\mathrm{a}^{2}$ of the original differential operators standing in the left-hand-sides of the differential equations of motion. Thus, expanding the differential and integral operators in powers of squares of the basic parameters constitutes an essential part of the theory. These expansions are also important in forming the equations satisfied by terms of higher orders in the development of $\mathrm{c}$ and $\mathrm{g}$. Applying the expansion of the double differential operator to the terms proportional to e or, , and suppressing the secular terms in the parallax and the $z$-coordinate, can yield the equations for terms of the expansion of $\mathrm{c}$ and $\mathrm{g}$.

The process of integration in the Hill-Brown theory is connected with the literal expansion of the coordinates, frequencies and operators in powers of basic parameters. However, the literal expansion in $\mathrm{m}$ in the Hill-Brown theory is not as essential as in the theories based on removal of short period terms by means of canonical transformations. Thus, to simplify the computations, we replace the problem of analytical convergence by the problem of numerical convergence and suggest, together with Brown, substituting the numerical value of $\mathrm{m}$ from the outset for any particular satellite.

In solving the differential equations we must derive the expansions of $w^{3}$, (the cube of the parallax) and $\rho^{2}$, (the square of the distance of the satellite from the planet) in powers and products of the perturbations of parallax $w=1 / \mathrm{w}$. This is accomplished by expanding the corresponding Taylor operator in powers and products of the perturbations in $w$ and into series in so-called Faa-de-Bruno differential operators (Faa-de-Bruno, 1855). These last operators are associated with the formation of higher derivatives of a function of a function. The Taylor operator thus expande is then applied to $\mathrm{w}^{3}$ and $\mathrm{x}^{2}$. The differential, integral and Taylor operators in their expanded form, together with the extraction operator, constitute a complete set of operators to be used in deriving a literal theory in rectangular coordinates by iteration.

The extraction operator performs the extraction of the terms of the desired type from the right-hand sides of the differential equations of motion. It is imbedded into analytical programming languages. In the application to the satellites of outer planets, series will, normally, never be very 
long. The number of numerically significant terms with a given characteristic will be of order few tens and the number of iterations will not be much larger than five.

\section{THE BASIC DIFFERENTIAL EQUATIONS}

The starting point of our exposition is Hill's system of differential equations of motion of the satellite:

where

$$
\begin{gathered}
D^{2} u+2 m D u+\frac{3}{2} m^{2}(u+s)-\kappa w^{3} u=-\frac{\partial \Omega}{\partial s}, \\
D^{2} s-2 m D s+\frac{3}{2} m^{2}(u+s)-\kappa w^{3} s=-\frac{\partial \Omega}{\partial u} \\
\left(D^{2}-m^{2}\right) z-\kappa w^{3} z=-\frac{1}{2} \frac{\partial \Omega}{\partial z} \\
\kappa=\frac{f(E+M)}{\left(n-n^{\prime}\right)^{2}}=(1+m)^{2} a^{3} .
\end{gathered}
$$

The form of expansion of the coordinates is

$$
\begin{aligned}
& u \zeta^{-1}, s \zeta, z \sqrt{1}=a \sum F(m) e^{p} e^{\prime p^{\prime}} \gamma^{q} a^{r} \zeta^{2 i+j c+j^{\prime} m+k g,} \\
& p=|j|+2 p_{1}, p^{\prime}=\left|j^{\prime}\right|+2 p_{1}^{\prime}, q=|k|+2 q_{1}, r=|2 i|+2 r_{1},
\end{aligned}
$$

where $p_{1}, p_{1}^{\prime}, q_{1}, r_{1}$ are positive integers. The monomial $\lambda=e^{p} e^{\prime p^{\prime}} y^{q} a^{r}$ is the characteristic of the term in the expansion; $p+p^{\prime}+q+r$ is its order. The linear factor a is defined in such a manner that the constant term in the variational part of $u r-1 / a$ is equal to 1 .

We shall reduce the differential equations (1) - (3) to a form suitable for the computation of terms with the prescribed characteristic using the iterative procedure. This form is convenient for programming and computation on electronic machines. We introduce dimensionless coordinates, replacing $u, s, z$ by ua, sa , a z, respectively. Equations (1) - (3) preserve their form after this change in the meaning of notations. The definition of $\kappa$, however, will not be as in (4), but will become

$$
k=(1+m)^{2} \frac{a^{3}}{a^{3}} .
$$

Substituting the variational solution

$$
\begin{aligned}
& u_{0}=\zeta\left[1+\mathrm{m}^{2} \mathrm{P}\left(\zeta^{2}\right)\right], \\
& \mathrm{s}_{0}=\zeta^{-1}\left[1+\mathrm{m}^{2} \mathrm{P}\left(\zeta^{-2}\right)\right],
\end{aligned}
$$


where $P\left(\zeta^{2}\right)$ is the Laurent series in $\zeta^{2}$, into $(1)-(2)$, we deduce for the new value of $\kappa$ defined by $(5)$,

$$
\kappa=\kappa_{0}+\delta \kappa,
$$

where

$$
\kappa_{0}=1+2 \mathrm{~m}+\frac{3}{2} \mathrm{~m}^{2}
$$

and $\delta \kappa$ is of the fourth order relative to $\mathrm{m}$. We obtain the numerical value of $\delta \kappa$ by iteration, during the process of deriving the variational solution $u_{0}, s_{0}$. The condition now must be imposed that the constant terms in $u_{0} \zeta^{-1}$ and $s_{0} \zeta^{+1}$ are equal to 1 .

We introduce a set of partial differential operators,

$$
\Lambda, \Lambda^{\prime}, \Gamma, \text { and } r \text {, }
$$

acting on the expression of the type

$$
\phi=\zeta^{2 i+j c+k g+j} m
$$

in the following manner:

$$
\Lambda \phi=\mathbf{j} \phi, \Lambda^{\prime} \phi=\mathbf{j}^{\prime} \phi, \Gamma \phi=\mathrm{k} \phi, \Upsilon \phi=2 \mathbf{i} \phi .
$$

Evidently

$$
D=Y+c \Lambda+g \Gamma+m \Lambda^{\prime}
$$

The expansions

$$
c=c_{0}+c_{2}+c_{4}+\cdots, g=g_{0}+g_{2}+g_{4}+\cdots \cdot
$$

where $c_{2 j}$ and $g_{2 j}$ are the polynomials of the $j$ th order in squares of the basic parameters $e$, $e^{\prime}$, $\gamma$, and $a$, are not initially known and must be determined step by step. Consequently, neither the operator $\mathrm{D}$ nor its inverse $\mathrm{D}^{-1}$ can be used. The theory of Hill and Brown is formulated so that not $\mathrm{D}$ and $\mathrm{D}^{-1}$, but the operator

$$
D_{0}=\Upsilon+c_{0} \Lambda+g_{0} \Gamma+m \Lambda^{\prime}
$$

and its inverse are used throughout. We have the expansion

$$
\mathrm{D}=\mathrm{D}_{0}+\mathrm{D}_{2}+\mathrm{D}_{4}+\cdots \cdot
$$


where

$$
\begin{aligned}
& \mathrm{D}_{2 \mathrm{~s}}=\mathrm{c}_{2 \mathrm{~s}} \Lambda+\mathrm{g}_{2 \mathrm{~s}} \Gamma, \\
& \mathrm{s}=1,2,3, \ldots .
\end{aligned}
$$

We have

$$
\begin{gathered}
D_{0} \phi=\left(2 i+c_{0} j+g_{0} k+m j^{\prime}\right) \phi, \\
D_{2 s} \phi=\left(j c_{2 s}+k g_{2 s}\right) \phi, \\
D_{0}^{-1} \phi=\left(2 i+c_{0} j+g_{0} k+m j^{\prime}\right)^{-1} \phi .
\end{gathered}
$$

Following Andoyer (1926) we set $u=\zeta(1+p), s=\zeta^{-1}(1+q)$. Equations (1) and (2) then become

$$
\begin{gathered}
{\left[D^{2}+2(1+m) D+\kappa_{0}\right] p+\kappa_{0}-\kappa w^{3}(1+p)+\frac{3}{2} m^{2} y^{-2}(1+q)=-\frac{\partial \Omega}{\partial q}} \\
{\left[D^{2}-2(1+m) D+\kappa_{0}\right] q+\kappa_{0}-\kappa w^{3}(1+q)+\frac{3}{2} m^{2} \zeta^{2}(1+p)=-\frac{\partial \Omega}{\partial p},} \\
\kappa_{0}=1+2 m+\frac{3}{2} m^{2} .
\end{gathered}
$$

We rewrite them in a form more convenient for applying the process of iteration, i.e.,

$$
\begin{aligned}
& {\left[\mathrm{D}^{2}+2(1+\mathrm{m}) \mathrm{D}+\frac{3}{2} \kappa_{0}\right] \mathrm{p}+\frac{3}{2} \kappa_{0} \mathrm{q}=\mathrm{P}-\frac{\partial \Omega}{\partial \mathrm{q}},} \\
& {\left[\mathrm{D}^{2}-2(1+\mathrm{m}) \mathrm{D}+\frac{3}{2} \kappa_{0}\right] \mathrm{q}+\frac{3}{2} \kappa_{0} \mathrm{P}=\mathrm{Q}-\frac{\partial \Omega}{\partial \mathrm{p}},}
\end{aligned}
$$

where we set

$$
\begin{gathered}
P=-\frac{3}{2} m^{2} \zeta^{-2}(1+q)+\kappa_{0}\left[\left(w^{3}-1\right)(1+p)+\frac{3}{2}(p+q)\right]+w^{3}(1+p) \delta \kappa \\
Q=-\frac{3}{2} m^{2} \zeta^{2}(1+p)+\kappa_{0}\left[\left(w^{3}-1\right)(1+q)+\frac{3}{2}(p+q)\right]+w^{3}(1+q) \delta \kappa
\end{gathered}
$$


The quantities $p$ and $q$ are complex conjugates. Consequently, only one of the equations (10) and (11) can be considered. We can set $\Omega=0$ if we are interested only in terms depending upon e and $\gamma$.

Taking

$$
p^{2}-(1+p)(1+q)+z^{2}
$$

into account, we can put (10) into the form

$$
\begin{aligned}
P=-\frac{3}{2} m^{2} \zeta^{-2}(1+q) & \\
& +0\left[\left(\frac{3}{2} \dot{r}^{2}+w^{3}-\frac{5}{2}\right)-\left(w^{3}-1\right) p-\frac{3}{2}\left(p q+z^{2}\right)\right] \\
& +w^{3}(1+p) \delta \kappa,
\end{aligned}
$$

convenient for using the iterative procedure. We now split $p, q, w$ and $P$ into the variational part, and the part which contains all terms with positive characteristics, and set

$$
\begin{gathered}
\mathrm{p}=\xi_{0}-\xi, \mathrm{q}=\eta_{0}+\eta, \mathrm{w}=\mathrm{w}_{0}+\mathrm{w}_{0}^{3} x \\
\mathrm{P}=\left(\Xi_{0}+\delta k\right)+\Xi .
\end{gathered}
$$

The zero-subscript refers to the variational part. From (14) we deduce

$$
\begin{gathered}
\rho^{2}=\rho_{0}^{2}-2 x+A, \\
w^{3}=w_{0}^{3}+3 w_{0}^{5} x+B,
\end{gathered}
$$

where A and B are of order not smaller than two. When the terms with the given characteristic are to be determined, then the terms with the same characteristic as contained in A and B must always represent the "cross-effects" between the terms of some lower characteristics, namely, between the terms which were computed at a previous step.

The analytical representation of these cross-effects will be discussed later in this exposition. From (12), (14) and (16) we deduce the relation

$$
\mathrm{A}=2 x+\left(1+\eta_{0}\right) \xi+\left(1+\xi_{0}\right) \eta+\left(\xi \eta+z^{2}\right) .
$$


Substituting (14) into (13) we obtain

$$
\begin{gathered}
\Xi_{0}=-\frac{3}{2} m^{2} \zeta^{-2}\left(1+\eta_{0}\right) \\
+\kappa_{0}\left[\left(\frac{3}{2} \rho_{0}^{2}+w_{0}^{3}-\frac{5}{2}\right)+\left(w_{0}^{3}-1\right) \xi_{0}-\frac{3}{2} \xi_{0} \eta_{0}\right] \\
+\left[\left(1+\xi_{0}\right) w_{0}^{3}-1\right] \delta \kappa .
\end{gathered}
$$

or, decomposing into factors,

$$
\begin{aligned}
& \Xi_{0}=-\frac{3}{2} m^{2} \zeta^{-2}\left(1+\eta_{0}\right) \\
& -\kappa_{0}\left\{\left(w_{0}-1\right)^{2}\left[3 r_{0}^{2}\left(\frac{1}{2}+w_{0}\right)+\left(2-w_{0}\right)\right]\right. \\
& \left.+\left(w_{0}^{3}-1\right) \xi_{0}-\frac{3}{2} \xi_{0} \eta_{0}\right\} \\
& +\left[w_{0}^{3}\left(1-\xi_{0}\right)-1\right] \delta \kappa
\end{aligned}
$$

This representation of $\Xi_{0}$ is suitable for the iterative procedure, because the three terms on the right-hand side are of second, fourth, and sixth order in m, respectively. The differential equations for $\xi_{0}$ and $\eta_{0}$ are

$$
\begin{aligned}
& {\left[D_{0}^{2}+2(1+m) D_{0}+\frac{3}{2} \kappa_{0}\right] \xi_{0}-\frac{3}{2} \kappa_{0} \eta_{0}=\Xi_{0}-\delta \kappa,} \\
& {\left[D_{0}^{2}-2(1+m) D_{0}+\frac{3}{2} \kappa_{0}\right] \eta_{0}+\frac{3}{2} \kappa_{0} \xi_{0}=H_{0}+\delta \kappa,}
\end{aligned}
$$

where

$$
\mathrm{H}_{0}=\bar{\Xi}_{0} \text {. }
$$


We actually need only one of these equations. They are a paraphrase of the differential equations

$$
\begin{aligned}
& {\left[D_{0}^{2}+2(1+m) D_{0}+\kappa_{0}\right] \xi_{0}+\kappa_{0}-\kappa w_{0}^{3}\left(1+\xi_{0}\right)+\frac{3}{2} \mathrm{~m}^{2} \zeta^{-2}\left(1+\eta_{0}\right)=0,} \\
& {\left[D_{0}^{2}-2(1+m) D_{0}+\kappa_{0}\right] \eta_{0}+\kappa_{0}-\kappa w_{0}^{3}\left(1+\eta_{0}\right)+\frac{3}{2} m^{2} \zeta^{2}\left(1+\xi_{0}\right)=0,}
\end{aligned}
$$

easily obtainable from the general form (6) and (7), but (19) and (20) are in a form more convenient for using an analytical programming language.

In a similar manner we deduce from (13), taking (12), (16) and (17) into account, that

$$
\begin{aligned}
\Xi & =\left(\kappa \mathrm{w}_{0}^{3}-\kappa_{0}-\frac{3}{2} \kappa_{0} \eta_{0}\right) \xi \\
& -\frac{3}{2}\left(\mathrm{~m}^{2} \zeta^{-2}+\kappa_{0} \xi_{0}\right) \eta \\
& +3\left[\kappa \mathrm{w}_{0}^{5}\left(1+\xi_{0}\right)-\kappa_{0}\right] x \\
& +\kappa\left[3 \mathrm{w}_{0}^{5} \xi x+\mathrm{B}\left(1+\xi_{0}+\xi\right)\right]+\frac{3}{2} \kappa_{0}\left[\mathrm{~A}-\left(\xi \eta+\mathrm{z}^{2}\right)\right] .
\end{aligned}
$$

Eliminating $x$ from the last equation by means of (18), we obtain $\Xi$ in the form suitable for actual computations,

$$
\Xi=\mathbf{M} \xi+\mathbf{N} \eta+\mathbf{S},
$$

where we set

$$
\begin{aligned}
M & \frac{1}{2}\left(\kappa_{0}-\kappa w_{0}^{3}\right) \\
= & -\frac{1}{2}\left[\kappa\left(w_{0}^{3}-1\right)+\delta \kappa\right],
\end{aligned}
$$




$$
\begin{aligned}
& \mathbf{N}= \frac{3}{2}\left[\kappa_{0}-\kappa \mathrm{w}_{0}^{5}\left(1+\xi_{0}\right)^{2}-\mathrm{m}^{2} \zeta^{-2}\right] \\
&=-\frac{3}{2}\left\{\kappa\left[\mathrm{w}_{0}^{5}\left(1+\xi_{0}\right)^{2}-1\right]+\mathrm{m}^{2} \zeta^{-2}+\delta \kappa\right\}, \\
& \mathbf{S}=\frac{3}{2} \kappa \mathrm{w}_{0}^{5}\left(1+\xi_{0}\right)\left[\mathrm{A}-\left(\xi \eta+z^{2}\right)\right] \\
&+\kappa\left[3 \mathrm{w}_{0}^{5} \xi x+B\left(1+\xi_{0}+\xi\right)\right] .
\end{aligned}
$$

Taking into account (8), (13), (15) and (23) - (26), we conclude that the aggregate of all terms with positive characteristics satisfies the differential equation

$$
\left[\mathrm{D}^{2}+2(\mathbf{1}+\mathrm{m}) \mathrm{D}+\frac{3}{2} \kappa_{0}\right] \xi+\frac{3}{2} \kappa_{0} \eta=\mathrm{M} \xi+\mathrm{N} \eta+\left(\mathrm{S}-\frac{\partial \Omega}{\partial \eta}\right)
$$

We can rewrite the last equation in the form

$$
\begin{aligned}
& {\left[\mathrm{D}_{0}^{2}+2(1+\mathrm{m}) \mathrm{D}_{0}+\frac{3}{2} \kappa_{0}\right] \xi+\frac{3}{2} \kappa_{0} \eta=M \xi+\mathrm{N} \eta+\mathrm{S}-\frac{\partial \Omega}{\partial \eta}} \\
& -\left[2\left(D_{0}+1+\mathrm{m}\right) \delta \mathrm{D}+(\delta \mathrm{D})^{2}\right] \xi,
\end{aligned}
$$

where

$$
\begin{array}{r}
\delta D=D-D_{0}=\Lambda \delta c+\Gamma \delta g, \\
\delta c=c-c_{0}, \quad g=g-g_{0} .
\end{array}
$$

This form is suitable for computations and for deriving the terms with a given characteristic (or order), because the coefficients $M$ and $N$ contain $m^{2}$ as a factor.

In the process of deriving the terms with the prescribed characteristic $\lambda$, the corresponding parts contributed by $S$ and $\left[2\left(D_{0}+1+m\right) \delta D+(\delta D)^{2}\right] \xi$ represent the cross-action of terms with characteristics lower than $\lambda$. The expansion of $S$ starts with the second-order terms. The expression $2\left(D_{0}+1+m\right) \delta D \xi_{0}$ contains the terms $c_{2}, c_{4}, \ldots$ of the expansion of c linearly and is closely associated with the formation of the linear equations for their determination. The parts $\xi_{\mathrm{e}}$ and $\eta_{\mathrm{e}}$, which are proportional to the first power of e, satisfy the differential equation 


$$
\begin{aligned}
& {\left[\mathrm{D}_{0}^{2}+2(1+\mathrm{m}) \mathrm{D}_{0}+\frac{3}{2} \kappa_{0}\right] \xi_{\mathrm{e}}+\frac{3}{2} \kappa_{0} \eta_{\mathrm{e}}=\mathrm{M} \xi_{\mathrm{e}}+\mathrm{N} \eta_{\mathrm{e}},} \\
& {\left[\mathrm{D}_{0}^{2}-2(1+\mathrm{m}) \mathrm{D}_{0}+\frac{3}{2} \kappa_{0}\right] \eta_{\mathrm{e}}+\frac{3}{2} \kappa_{0} \xi_{\mathrm{e}}=\mathrm{M} \eta_{\mathrm{e}}+\mathrm{N} \xi_{\mathrm{e}} .}
\end{aligned}
$$

To derive the expansion of the parallax one can use the equation established by Andoyer (1926):

$$
\begin{aligned}
K w & +\left(m^{2}+\frac{1}{2} D^{2}\right) p^{2}-3 m^{2} z^{2} \\
& +\frac{3}{2} m^{2}\left(1+m D^{-1}\right) u^{2}+\frac{3}{2} m^{2}\left(1-m D^{-1}\right) s^{2}-C+\Phi=0,
\end{aligned}
$$

where

$$
\begin{aligned}
\Phi=\Omega & +\frac{1}{2}\left(u \frac{\partial \Omega}{\partial u}-s \frac{\partial \Omega}{\partial s}+z \frac{\partial \Omega}{\partial z}\right) \\
& +m D^{-1}\left(u \frac{\partial \Omega}{\partial u}-s \frac{\partial \Omega}{\partial s}-\frac{\partial \Omega}{\partial i \ell^{\prime}}\right)
\end{aligned}
$$

$c$, the constant of integration, is developable into a series

$$
\mathrm{C}=\mathrm{C}_{0}+\mathrm{C}_{2}+\mathrm{C}_{4}+\cdots \cdot
$$

where $C_{2 j}$ are homogeneous polynomials of $j$ th degree in $e^{2}, e^{\prime 2}, \gamma^{2}$ and $a^{2}$.

Substituting (14) into (29) we can split the differential equation into the equation for the variational part in parallax

$$
\begin{aligned}
\kappa w_{0} & +\left(m^{2}+\frac{1}{2} D_{0}^{2}\right) \rho_{0}^{2} \\
& +\frac{3}{2} m^{2}\left(1+m D_{0}^{-1}\right)\left[\zeta^{+2}\left(1+\xi_{0}\right)^{2}\right] \\
& +\frac{3}{2} m^{2}\left(1-m D_{0}^{-1}\right)\left[\zeta^{-2}\left(1+\eta_{0}\right)^{2}\right]=C_{0},
\end{aligned}
$$

and the differential equation for the aggregate of all terms with positive characteristics 


$$
\begin{aligned}
\left(D_{0}^{2}-\kappa\right. & \left.+2 m^{2}\right) \chi=\kappa\left(w_{0}^{3}-1\right) \chi \\
& +\left(m^{2}+\frac{1}{2} D^{2}\right) A-3 m^{2} z^{2} \\
& +3 m^{2}\left(1+m D^{-1}\right)\left[\zeta^{+2}\left(1+\xi_{0}+\frac{1}{2} \xi\right) \xi\right] \\
& +3 m^{2}\left(1-m D^{-1}\right)\left[\zeta^{-2}\left(1+\eta_{0}+\frac{1}{2} \eta\right) \eta\right]-\delta C+\Phi .
\end{aligned}
$$

We do not follow in the footsteps of Andoyer and do not use the upper equation for the determination of $x$. The expansion of $x$ can be derived from(18) directly, without additional integration. We suggest, however, the use of the differential equation for the effects proportional to e,

$$
\begin{aligned}
\left(D_{0}^{2}-\kappa\right. & \left.+2 m^{2}\right) x_{e}=\kappa\left(w_{0}^{3}-1\right) x_{e} \\
& +3 m^{2}\left(1+m D_{0}^{-1}\right)\left[\zeta^{+2}\left(1+\xi_{0}\right) \xi_{e}\right] \\
& +3 m^{2}\left(1-m D_{0}^{-1}\right)\left[\zeta^{-2}\left(1+\eta_{0}\right) \eta_{e}\right],
\end{aligned}
$$

either to derive the value of $c_{0}$, if one prefers not to use an analytic equivalent to Hill's determinantal equation, or to check the expansions if necessary.

We can expand $x$ into a series

$$
x=x_{1}+x_{2}+x_{3}+\cdots
$$

where $x_{k}$ are homogeneous polynomials of kth degree, in e, $\mathbf{e}^{\prime}, \gamma$ and $a$, with the coefficients which are power series in $\zeta, \zeta^{\mathrm{c}}, \zeta^{\mathrm{g}}$ and $\zeta^{\mathrm{m}}$. We also need the expansion of $w^{3}$ and $\omega^{2}$ into a series in powers and products of $x_{1}, x_{2}, x_{3}, \ldots$.

Designating by $\mathrm{T}$ the Taylor operator

$$
T=\exp \left(w_{0}^{3} \times \frac{d}{d w_{0}}\right)
$$

we have

$$
\begin{aligned}
& w^{3}=T w_{0}^{3}, \\
& \rho^{2}=T \rho_{0}^{2} .
\end{aligned}
$$

The operator $T$ can be expanded into the series 


$$
\begin{aligned}
& \mathrm{T}=\mathrm{I}+\sum_{\mathrm{j}=1}^{+\infty} \sum_{\mathrm{k}=1}^{\mathrm{j}} \frac{1}{\mathrm{k} !} \mathrm{T}_{\mathrm{j}, \mathrm{k}}\left(\delta_{1}, \delta_{2}, \ldots, \delta_{k}\right) \\
& \mathrm{k}=1,2, \ldots \mathrm{j} \\
& \mathrm{j}=1,2,3, \ldots
\end{aligned}
$$

where $I$ is the identity operator and $T_{j, k}$ are Faa-de-Bruno (1855) differential operators. They are the homogeneous polynomials of kth degree relative to the operators

$$
\begin{aligned}
& \delta_{\mathrm{s}}=\mathrm{w}_{0}^{3} \chi_{\mathrm{s}} \frac{\mathrm{d}^{\mathrm{s}}}{\mathrm{d} \mathrm{w}_{0}^{\mathrm{s}}}, \\
& \mathrm{s}=1,2, \ldots,
\end{aligned}
$$

and satisfy the recursive relations

$$
T_{j, k}=\sum_{\sigma=1}^{j-k+1} \delta_{\sigma} T_{j-\sigma . k-1},
$$

or, in matrix form, the relation

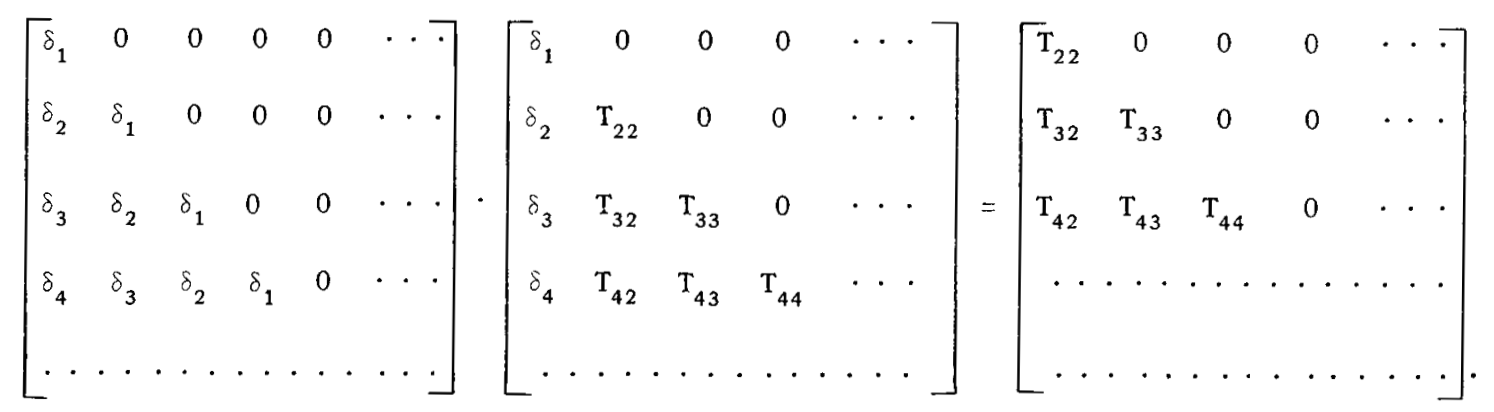

It follows from the homogeneity of $\mathbf{T}_{\mathbf{j}, k}$ that

$$
T_{j, k}\left(\delta_{1}, \delta_{2}, \ldots, \delta \kappa\right)=w_{0}^{3 k} T_{j, k}\left(x_{1}, x_{2}, \ldots, x_{k}\right) \frac{d^{k}}{d w_{0}^{k}}
$$

thus the symbols $\mathrm{T}_{j, k}$ shall designate the polynomials $\mathrm{T}_{\mathrm{j}, \mathrm{k}}\left(x_{1}, x_{2}, \ldots, x_{\mathrm{k}}\right)$. To compute them step by step we can use either (32) or (32') by replacing $\delta_{K}$ by $x_{k}$. By applying the general formula

$$
f(w)=T f\left(w_{0}\right)
$$


to the particular case

$$
f(w)=p^{2}, w^{3}
$$

and taking (16) and (17) into consideration, we obtain

$$
\begin{aligned}
& A=A_{2}+A_{3}+A_{4}+\cdots \\
& B=B_{2}+B_{3}+B_{4}+\cdots
\end{aligned}
$$

where

$$
A_{j}=+3 w_{0}^{2} T_{j, 2}-4 w_{0}^{4} T_{j, 3}+\cdots+(-1)^{j}(j+1) w_{0}^{2 j-2} T_{j, j}
$$

and

$$
\begin{gathered}
B_{2}=3 w_{0}^{7} T_{2,2} \\
B_{j}=3 w_{0}^{7} T_{j, 2}+w_{0}^{9} T_{j, 3}, \text { for } j \geqq 3 .
\end{gathered}
$$

The actual expansion of the polynomials $\mathbf{T}_{j, k}$ in powers of $\zeta, \zeta^{\mathrm{c}}, \zeta^{\mathrm{g}}$ and $\zeta^{\mathrm{m}}$, and powers of the basic parameters, should be performed on the electronic machine. However, for general orientation and for the purpose of checking, we give here the explicit form of these polynomials up to the eighth order:

$$
\begin{aligned}
& \mathrm{T}_{2,2}=x_{1}^{2}, \\
& \mathrm{~T}_{3,2}=2 x_{1} x_{2}, \\
& \mathrm{~T}_{3,3}=x_{1}^{3}, \\
& \mathrm{~T}_{4,2}=2 x_{1} x_{3}+x_{2}^{2}, \\
& \mathrm{~T}_{4,3}=3 x_{1}^{2} x_{2}, \\
& \mathrm{~T}_{4,4}=x_{1}^{4}, \\
& \mathrm{~T}_{5,2}=2 x_{1} x_{4}+2 x_{2} x_{3}, \\
& \mathrm{~T}_{5,3}=3 x_{1}^{2} x_{3}+3 x_{1} x_{2}^{2}, \\
& \mathrm{~T}_{5,4}=4 x_{1}^{3} x_{2}, \\
& \mathrm{~T}_{5,5}=x_{1}^{5}, \\
& \mathrm{~T}_{6,2}=2 x_{1} x_{5}+2 x_{2} x_{4}+x_{3}^{2}, \\
& \mathrm{~T}_{6,3}=3 x_{1}^{2} x_{4}+6 x_{1} x_{2} x_{3}+x_{2}^{3}, \\
& \mathrm{~T}_{6,4}=4 x_{1}^{3} x_{3}+6 x_{1}^{2} x_{2}^{2}, \\
& \mathrm{~T}_{6,5}=5 x_{1}^{4} x_{2}, \\
& \mathrm{~T}_{6,6}=x_{1}^{6},
\end{aligned}
$$




$$
\begin{aligned}
& \mathrm{T}_{7,2}=2 x_{1} x_{6}+2 x_{2} x_{5}+2 x_{3} x_{4}, \\
& \mathrm{~T}_{7,3}=3 x_{1}^{2} x_{5}+6 x_{1} x_{2} x_{4}+3 x_{1} x_{3}^{2}+3 x_{2}^{2} x_{3}, \\
& \mathrm{~T}_{7.4}=4 x_{1}^{3} x_{4}+12 x_{1}^{2} x_{2} x_{3}+4 x_{1} x_{2}^{3}, \\
& \mathrm{~T}_{7.5}=5 x_{1}^{4} x_{3}-10 x_{1}^{3} x_{2}^{2}, \\
& \mathrm{~T}_{7.6}=6 x_{1}^{5} x_{2}, \\
& \mathrm{~T}_{7,7}=x_{1}^{7}, \\
& \mathrm{~T}_{8,2}=2 x_{1} x_{7}+2 x_{2} x_{6}+2 x_{3} x_{5}+x_{4}^{2}, \\
& \mathrm{~T}_{8,3}=3 x_{1}^{2} x_{6}+6 x_{1} x_{2} x_{5}+6 x_{1} x_{3} x_{4}+3 x_{2}^{2} x_{4}+3 x_{2} x_{3}^{2}, \\
& \mathrm{~T}_{8,4}=4 x_{1}^{3} x_{5}+12 x_{1}^{2} x_{2} x_{4}+6 x_{1}^{2} x_{3}^{2}+12 x_{1} x_{2}^{2} x_{3}+x_{2}^{4} \\
& \mathrm{~T}_{8,5}=5 x_{1}^{4} x_{4}+20 x_{1}^{3} x_{2} x_{3}+10 x_{1}^{2} x_{2}^{3}, \\
& \mathrm{~T}_{8,6}=6 x_{1}^{5} x_{3}+15 x_{1}^{4} x_{2}^{2}, \\
& \mathrm{~T}_{8,7}=7 x_{1}^{6} x_{2}, \\
& \mathrm{~T}_{8,8}=x_{1}^{8} .
\end{aligned}
$$

\section{VARIATIONAL SOLUTION}

We derive Hill's variational solution of the lunar theory by applying the method of iteration directly to the system

$$
\begin{gathered}
{\left[\left(D_{0}+1+m\right)^{2}+\frac{1}{2}\left(\kappa_{0}+m^{2}\right)\right] \xi_{0}+\frac{3}{2} \kappa_{0} \eta_{0}=\Xi_{0}+\delta \kappa,} \\
\kappa w_{0}=-R_{0}+C_{0},
\end{gathered}
$$

where

$$
\begin{aligned}
\Xi_{0}= & -\frac{3}{2} m^{2} \zeta^{-2}\left(1+\eta_{0}\right) \\
& +\kappa_{0}\left\{\left(w_{0}-1\right)^{2}\left[3 \rho_{0}^{2}\left(w_{0}+\frac{1}{2}\right)+\left(w_{0}+2\right)\right]\right. \\
& \left.+\left(w_{0}^{3}-1\right) \xi_{0}-\frac{3}{2} \xi_{0} \eta_{0}\right\} \\
& +\left[w_{0}^{3}\left(1+\xi_{0}\right)-1\right] \delta \kappa,
\end{aligned}
$$




$$
\begin{aligned}
R_{0}= & \left(m^{2}+\frac{1}{2} D_{0}^{2}\right) \rho_{0}^{2} \\
& +\frac{3}{2} m^{2}\left(1+m D_{0}^{-1}\right)\left[\zeta^{+2}\left(1+\xi_{0}\right)^{2}\right] \\
& +\frac{3}{2} m^{2}\left(1-m D_{0}^{-1}\right)\left[\zeta^{-2}\left(1+\eta_{0}\right)^{2}\right] .
\end{aligned}
$$

The values of $\xi_{0}, \eta_{0}, w_{0}$ and ${ }_{k}{ }_{k}$ from the previous iteration can be substituted into $\Xi_{0}$ and $R_{0}$. We start the iteration with some suitable approximation to $\varepsilon_{k}, w_{0}, \xi_{0}$ and $n_{0}$. In the absence of better information, we can even begin with the values

$$
\gamma \kappa=0, \xi_{0} \quad \eta_{0}-0, w_{0}-1 .
$$

Substituting the selected first approximation into $\left(19^{\prime}\right)$, we derive better values for $\curvearrowright \kappa$, $\eta_{0}$. We determine $\times<$ such that $\Xi_{0}$ does not contain a constant term. The expansion of $\Xi_{0}$ has the form

$$
\Xi_{0} \cdot \sum_{j=1}^{+\infty}\left(A_{+j} \zeta^{+2 \jmath}+A_{-j} \zeta^{-2 \jmath}\right)
$$

The expansions of $\%_{0}$ and $\eta_{0}$ are of similar type:

$$
\begin{aligned}
& =\sum_{j=1}^{+\infty}\left(a_{+}, \zeta^{+2 \jmath}+a_{-j} \zeta^{-2 \jmath}\right), \\
& \eta_{0}=\sum_{j=i}^{+r}\left(a_{-j} \zeta^{+2 \jmath}-a_{+j} \zeta^{-2 \jmath}\right) .
\end{aligned}
$$

Substituting (35) and (36) into (19'), we obtain the system of "linear" equations:

$$
\begin{aligned}
& {\left[4 j^{2}+4(1+m) j+\frac{3}{2} \kappa_{0}\right] a_{+j}+\frac{3}{2} \kappa_{0} a_{-j}=A_{+j},} \\
& +\frac{3}{2} \kappa_{0} a_{+j}+\left[4 j^{2}-4(1-m) j-\frac{3}{2} \kappa_{0}\right] a_{-j}-A_{-j} .
\end{aligned}
$$


We obtain from (37) and (38):

$$
\begin{aligned}
& a_{+j}=[j, 0] A_{+j}+(j, 0) A_{-j} \\
& a-j=(j, 0) A_{+j}+\{j, 0\} A_{-j},
\end{aligned}
$$

where

$$
\begin{gathered}
{[j, 0]=+\frac{(2 j-1-m)^{2}+\frac{1}{2}\left(\kappa_{0}+m^{2}\right)}{4 j^{2}\left(4 j^{2}-\kappa_{0}+2 m^{2}\right)},} \\
(j, 0)=-\frac{3}{2} \frac{\kappa_{0}}{4 j^{2}\left(4 j^{2}-\kappa_{0}+2 m^{2}\right)} \\
\{j, 0\}=-\frac{(2 j+1+m)^{2}+\frac{1}{2}\left(\kappa_{0}+m^{2}\right)}{4 j^{2}\left(4 j^{2}-\kappa_{0}+2 m^{2}\right)}-=[-j, 0] .
\end{gathered}
$$

The coefficients $\{j, 0\},(j, 0)$ and $\{j, 0\}$ remain the same from iteration to iteration. We substitute the values of $a, s_{0}$ and $\eta_{0}$ thus obtained into $\left(29^{\circ}\right)$ and derive $w_{0}$ without any integration. The value of the constant $C_{0}$ is obtained at each iteration from the condition

$$
\begin{gathered}
\mathrm{C}_{0}=\left(\kappa \mathrm{w}_{0}+\mathrm{R}_{0}\right)_{\zeta=1}, \\
\left.\mathrm{w}_{0}\right\}_{\zeta=1}=\left[\left(1-\bar{\xi}_{0}\right)^{-1}{ }^{\prime 2}\left(1+\eta_{0}\right)^{-1}\right]_{\zeta=1} .
\end{gathered}
$$

We proceed to higher approximations in the same manner, till we reach the convergence.

\section{THE TERMS DEPENDING UPON THE FIRST POWER OF THE ECCENTRICITY}

The computation of the first-order effects in e is intimately connected with the computation of $c_{0}$. We have already derived the system of differential equations

$$
\begin{aligned}
& {\left[\left(\mathrm{D}_{0}+1+\mathrm{m}\right)^{2}\right.}\left.+\frac{1}{2}\left(\kappa_{0}+\mathrm{m}^{2}\right)\right] \xi_{\mathrm{e}}+\frac{3}{2} \kappa_{0} \eta_{\mathrm{e}}-\mathrm{M} \xi_{\mathrm{e}}+\mathrm{N} \eta_{\mathrm{e}}, \\
&\left(\mathrm{D}_{0}^{2}-\alpha+2 \mathrm{~m}^{2}\right) x_{\mathrm{e}}=\kappa\left(\mathrm{w}_{0}^{3}-1\right) x_{\mathrm{e}} \\
&-3 \mathrm{~m}^{3}\left(1-\mathrm{m} \mathrm{D}_{0}^{-1}\right)\left[\zeta^{+2}\left(1+\zeta_{0}\right) \xi_{\mathrm{e}}\right] \\
&-3 \mathrm{~m}^{2}\left(1-\mathrm{mD}_{0}^{-1}\right)\left[\zeta^{-2}\left(1+\eta_{0}\right) \eta_{\mathrm{e}}\right],
\end{aligned}
$$




$$
\begin{gathered}
M=\frac{1}{2}\left(\kappa_{0}-\kappa w_{0}^{3}\right), \\
N=\frac{3}{2}\left[\kappa_{0}-\kappa w_{0}^{5}\left(1+\xi_{0}\right)^{2}-m^{2} \zeta^{-2}\right],
\end{gathered}
$$

which govern the perturbations proportional to e and are in a form suitable for solving by iteration. The relation

$$
2 x_{e}+\left(1+\eta_{0}\right) \check{\zeta}_{e}+\left(1+\check{5}_{0}\right) \eta_{e}=0
$$

can be used to determine $x_{\mathrm{e}}$ without any additional integration. This equation can serve for checking the computations and for deriving the value of $c_{0}$. At each iteration step, the differential equations for terms with the first power of e as a factor have the form:

$$
\begin{gathered}
{\left[\left(D_{0}+1+m\right)^{2}+\frac{1}{2}\left(\kappa_{0}+m^{2}\right)\right] \xi_{e}+\frac{3}{2} \kappa_{0} m_{0}-\sum_{1}\left(i_{+i} \zeta^{+2 i+c}+y_{-i} \zeta^{-21-c}\right),} \\
\left(D_{0}^{2}-\kappa+2 m^{2}\right) x_{e}=\sum_{1} \sigma_{i}\left(\zeta_{0}^{+2 i+c}+\zeta-2 i-c\right),
\end{gathered}
$$

and correspondingly, the solutions will be of a similar form

$$
\begin{aligned}
& \xi_{e}=\sum_{1}\left(\epsilon_{+i} \zeta^{2 i+c}+\epsilon_{-i}^{\prime} \zeta^{-21-c}\right), \\
& \eta_{e}=\sum_{1}\left(\epsilon_{-i}^{\prime} \zeta^{+2 i+c}+\epsilon_{+i} \zeta^{-2 i-c}\right), \\
& x_{e}=\sum_{1} \lambda_{i}\left(\zeta^{+2 i+c}+\zeta^{-2 i-c}\right) .
\end{aligned}
$$

Substituting these expressions into the differential equations and comparing the terms in both sides, we derive

$$
\begin{aligned}
& \epsilon_{i}=[i, \text { c }] \beta_{+i}+(i, c) \beta_{-i}^{\prime}, \\
& \epsilon_{-i}^{\prime}=(i, c) \beta_{+i}+\{i, c\} \beta_{-i}^{\prime},
\end{aligned}
$$


where we set

$$
\begin{gathered}
{[i, c]=\frac{\left(2 i+c_{0}-1-m\right)^{2}+\frac{1}{2}\left(\kappa_{0}+m^{2}\right)}{\left(2 i+c_{0}\right)^{2}\left(\left(2 i+c_{0}\right)^{2}-\kappa_{0}+2 m^{2}\right]},} \\
(i, c)=-\frac{3}{2} \frac{\kappa_{0}}{\left(2 i+c_{0}\right)^{2}\left[\left(2 i-c_{0}\right)^{2}-\kappa_{0}+2 m^{2}\right]}, \\
\{i, c\}=\frac{\left(2 i+c_{0}+1+m\right)^{2}+\frac{1}{2}\left(\kappa_{0}+m^{2}\right)}{\left(2 i+c_{0}\right)^{2}\left[\left(2 i+c_{0}\right)^{2}-\kappa_{0}+2 m^{2}\right]} .
\end{gathered}
$$

At each cycle, the value of $c_{0}$ is obtained from the condition

$$
c_{0}^{2}=\kappa-2 m^{2}+\frac{\sigma_{0}}{\lambda_{0}} .
$$

The term $\sigma_{0} /{ }_{0}$ is of the third order in $\mathrm{m}$. The largest portion of it is contributed by the evection in $\xi_{e}$ and $\eta_{e}$. The remaining, much smaller, part is contributed by smaller terms in $\xi_{e}$ and $\eta_{e}$. Their order in $m$ is not smaller than four. The deriving of $\epsilon_{0}$ and $\epsilon_{0}^{\prime}$ requires, as in the classical approach, a special device, because a spurious small divisor appears in (45) - (47) for $\mathrm{i}=0$.

In the method based on the iterative procedure, the values of $\epsilon_{0}$ and $\epsilon_{0}^{\prime}$ can be derived from the equations

$$
\begin{aligned}
& {\left[\left(c_{0}-1+m\right)^{2}+\frac{1}{2}\left(\kappa_{0}+m^{2}\right)\right] \epsilon_{0}+\frac{3}{2} \kappa_{0} \epsilon_{\theta}^{\prime}=\xi_{0},} \\
& {\left[\left(c_{0}-1-m\right)^{2}+\frac{1}{2}\left(\kappa_{0}+m^{2}\right)\right] \epsilon_{0}^{\prime}+\frac{3}{2} \kappa_{0} \epsilon_{0}=\beta_{0}^{\prime},}
\end{aligned}
$$

which follow directly from (41), and combined with the additional condition

$$
\epsilon_{0}-\epsilon_{0}^{\prime}=1
$$

which we can borrow from the Hill-Brown exposition. In fact, it is equivalent to the definition of the mean orbital eccentricity of the satellite. Equations (48) - (50) are compatible and their determinant equals zero. We deduce from the last three equations

$$
\epsilon_{0}=\left(\beta_{0}+\frac{3}{2} \kappa_{0}\right) /\left[\left(c_{0}+1+m\right)^{2}+2 \kappa_{0}+\frac{1}{2} m^{2}\right],
$$




$$
\epsilon_{0}^{\prime}=\left(\beta_{0}^{\prime}-\frac{3}{2} \kappa_{0}\right) /\left[\left(c_{0}-1-m\right)^{2}+2 \kappa_{0}+\frac{1}{2} m^{2}\right] .
$$

The substitution of these values into (50) can serve as a check of the speed of convergence of the iterative process, and of the accuracy of computations. In applying the present theory to the motion of the seventh satellite of Jupiter, we found that the iterative procedure is rapidly convergent, even when we start with such a crude approximation as

$$
\begin{aligned}
& \xi_{e}=e\left(+\frac{1}{4} \zeta^{+c}-\frac{3}{4} \zeta^{-c}-\frac{45}{32} m \zeta^{-2+c}+\frac{15}{32} m \zeta^{+2-}\right), \\
& \eta_{e}=e\left(\frac{1}{4} \zeta^{-c}-\frac{3}{4} \zeta^{+c}-\frac{45}{32} m \zeta^{+2-c}-\frac{15}{32} m \zeta^{-2+c}\right) .
\end{aligned}
$$

Only a few iterations are necessary to obtain $\iota_{e}, r_{e}, \gamma_{e}$ and $c_{0}$ accurate to $10^{-9}$, which seems to be the standard accuracy in the expansion of the general theories of satellites of outer planets.

If the correct value of $c_{0}$ is initially given, then coefficients (45) - (47) remain the same from iteration to iteration. We can dispense with the differential equation for $x_{e}$, and the iterative process is considerably shortened. The correct value of $c_{0}$ can be obtained using Andoyer's (1926) equation, which is equivalent to Hill's equation but provides a fast convergence for $c_{0}$ and is convenient for programming. We follow Hill in the computation of auxiliary quantities, and set:

$$
\begin{aligned}
& \left.x(1-D) \operatorname{ti}\left(1-\frac{5}{0}\right)\right\} \\
& \therefore=(1-D) ! y^{-1}\left(1-n_{0}\right): \\
& \mathrm{A}^{\prime}=(1-x)^{-1} \mathrm{D} \cdot \mathrm{x}, \quad \mathrm{B}^{\prime}-\ddot{A}^{\prime}-(1-)^{-1} \mathrm{D} \cdots, \\
& P^{\prime}-1 \cdot \frac{1}{2}\left(A^{\prime}-B^{\prime}\right)+m, \\
& Q^{\prime}-\frac{1}{2}\left(A^{\prime}-B^{\prime}\right), \\
& S--\left(\kappa w_{0}^{3}+m^{2}\right)-\left(2 P^{\prime 2}-Q^{\prime 2}-D Q^{\prime}\right) \\
& \sum_{-\infty}^{+r} S_{2 j} \gamma_{0}^{2 j}, \\
& S_{-2 j}-S_{+2 j}
\end{aligned}
$$


Introducing the notations

$$
\begin{gathered}
c_{0}^{2}=S_{0}+n_{0}, \\
\mu_{j}=\left(j^{2}+2 j c_{0}+n_{0}\right)^{-1},
\end{gathered}
$$

we write the Andoyer expansion in the form

$$
\begin{aligned}
& \mathrm{n}_{0}=\mathrm{S}_{2}^{2}\left(\mu_{+2}+\mu_{-2}\right) \\
& +\mathrm{S}_{2}^{4}\left(\mu_{+2}^{2} \mu_{+4}+\mu_{-2}^{2} \mu_{-4}\right) \\
& +2 \mathrm{~S}_{4} \mathrm{~S}_{2}^{2}\left(\mu_{+2} \mu_{+4}+\mu_{-2} \mu_{-4}+\mu_{+2} \mu_{-2}\right) \\
& +S_{4}^{2}\left(\mu_{+4}+\mu_{-4}\right) \\
& +\mathrm{S}_{2}^{6}\left(\mu_{+2} \mu_{+4}^{2} \mu_{+6}-\mu_{-2}^{2} \mu_{-4}^{2} \mu_{-6}-\mu_{+2}^{3} \mu_{+4}^{2}+\mu_{-2}^{3} \mu_{-4}^{2}\right) \\
& +2 \mathrm{~S}_{4} \mathrm{~S}_{2}^{4}\left(\mu_{+2} \mu_{+4}^{2} \mu_{+6}+\mu_{-2} \mu_{-4}^{2} \mu_{-6}+\cdots+2 \alpha_{+4} \mu_{+6}\right. \\
& -\mu_{-2}^{2} \mu_{-4} \mu_{-6}+\mu_{+2}^{2} \mu^{2}+4+\mu_{-2}^{2} \mu_{-4}^{2} \\
& \left.\cdots+2{ }^{2} \mu_{-2} \mu+4-\mu_{+2} u_{-2}^{2} \mu_{-4}\right) \\
& -2 \mathrm{~S}_{6} \mathrm{~S}_{2}^{3}\left(\mu_{+2} \mu_{+4} \mu_{+6}+\mu_{-2} \mu_{-4} \mu_{-6}+\mu_{+2} \mu_{-2} \mu_{+4}-\mu_{+2} \mu_{-2} \mu_{-4}\right) \\
& -S_{4}^{2} S_{2}^{2}\left(\mu_{+4}^{2} \mu_{+6}+\mu_{-4}^{2} \mu_{-6} \cdot 2 \mu_{+2} \mu_{+4} \mu_{+6}+2 \mu_{-2} \mu_{-4} \mu_{-6}\right. \\
& +\mu_{+2}^{2} \mu_{+6}+\mu_{-2}^{2} \mu_{-6}-\mu_{+2} \mu_{+4}^{2}-\mu_{-2} \mu_{-4}^{2}+\mu_{+2} \mu_{-2} \mu_{+4} \\
& \text { - } \left.2{ }^{\prime}+2 \mu_{-2} \mu_{-4} \cdot u^{2}+2 \mu_{-2} \cdots+2 \mu_{-2}^{2}\right)
\end{aligned}
$$

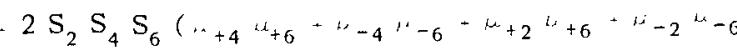

$$
\begin{aligned}
& \left.+u_{+2} u_{-4}-u_{-2} u_{+4}\right)+S_{6}^{2}\left(u_{+6}-u_{-6}\right)+\ldots
\end{aligned}
$$

The Andoyer expansion is convenient for programming and for computing $n_{0}$ and $c_{0}$ by iteration. It can be easily extended (Andoyer, 1926), if necessary. We tested it on Jupiter VII and found that four iterations were necessary to derive the value of $c_{0}$ accurate to $10^{-9}$.

\section{HIGHER ORDER TERMS IN $\xi$ AND $\eta$}

At this point we change the notations and replace $\xi, \cdot$ and $z$ by $\xi_{1}+\xi, \nu_{1}+\cdot$ and $z_{1}+\sigma$, respectively. As before, $\overline{\check{z}}_{1}, \gamma_{t_{1}}$ and $z_{1}$ designate the totality of effects of the first order,

$$
\begin{gathered}
\xi_{1}=z_{e}+\xi_{c}+\xi_{a}, \\
\eta_{1}=r_{e_{e}}-r_{e}+\eta_{x}, \\
z_{1}=z_{\gamma},
\end{gathered}
$$


and $\xi, \eta$ and $s$ from now on designate the effects of higher orders, starting from the second,

$$
\xi=\xi_{2}+\xi_{3}+\cdots, \eta=\eta_{2}+\eta_{3}+\cdots, s=z-z_{1}=z_{2}+z_{3}+\ldots
$$

From (27) we obtain

$$
\left[\left(D_{0}+1+\mathrm{m}\right)^{2}+\frac{1}{2}\left(\kappa_{0}+\mathrm{m}^{2}\right)\right] \xi_{1}+\frac{3}{2} \kappa_{0} \eta_{1}=\mathrm{M} \check{\zeta_{1}}+\mathrm{N} \eta_{1}-\left(\frac{\partial \Omega}{\partial \eta_{1}}\right)_{1}
$$

where $-(\partial \Omega / \partial \eta)_{1}$ are the first-order terms in $-\partial \Omega / \partial \eta$. They contain either $\mathrm{e}^{\prime}$ or a as a factor. Taking into account

$$
\Gamma \xi_{e}-\Lambda \xi_{e^{\prime}}=\Gamma \xi_{e^{\prime}}=\Lambda \xi_{a}=\Gamma \xi_{z}=0, \Lambda^{2} \xi_{e}=\xi_{e^{\prime}}
$$

we have for the effects of higher orders

$$
\begin{aligned}
{\left[\left(\mathrm{D}_{0}+1+\mathrm{m}\right)^{2}+\right.} & \left.\frac{1}{2}\left(\kappa_{0}+\mathrm{m}^{2}\right)\right] \xi+\frac{3}{2} \kappa_{0} \eta=\mathrm{M} \xi+\mathrm{N} r_{j}+\mathrm{F} \\
& -2 \circ \mathrm{c}\left(\mathrm{D}_{0}+1+\mathrm{m}\right) \Lambda \xi_{\mathrm{e}}
\end{aligned}
$$

where

$$
\mathrm{F}=-\frac{\partial \Omega}{\partial \eta}+\left(\frac{\partial \Omega}{\partial \eta}\right)_{1}-\left[2\left(\mathrm{D}_{0}+1+\mathrm{m}\right) \delta \mathrm{D}+(\delta \mathrm{D})^{2}\right] \xi-\xi_{\mathrm{e}}(\delta \mathrm{c})^{2}+\mathrm{S}
$$

This last expression represents the cross-action of terms of lower characteristics as compared to the characteristics of terms to be determined. We now introduce averaging operator $\mathfrak{m}$, which separates the constant terms from an expansion. Thus

$$
\mathfrak{M}\left\{\sum \mathrm{A}_{\mathrm{i}, j, j^{\prime}, \mathrm{k}} \zeta^{2 \mathrm{i}+\mathrm{je}+\mathrm{j}^{\prime} \mathrm{m}+\mathrm{kg}}\right\}=\mathrm{A}_{0,0,0,0^{-}}
$$

Multiplying both sides of (51) by $\eta_{e}$ and both sides of $\left(28^{\prime}\right)$ by $\xi$ and subtracting the results, we have

$$
\begin{gathered}
\mathrm{D}_{0}\left(\eta_{\mathrm{e}} \mathrm{D}_{0} \xi-\xi \mathrm{D}_{0} \eta_{\mathrm{e}}\right)+2(1+\mathrm{m})\left(\eta_{\mathrm{e}} \mathrm{D} \xi+\xi \mathrm{D}_{0} \eta_{\mathrm{e}}\right) \\
+\left(\frac{3}{2} \kappa_{0}-\mathrm{N}\right)\left(\eta \eta_{\mathrm{e}}-\xi \xi_{\mathrm{e}}\right)=\eta_{\mathrm{e}} \mathrm{F}-2 \eta_{\mathrm{e}} \delta \mathrm{c}\left(\mathrm{D}_{0}+1+\mathrm{m}\right) \Lambda \xi_{\mathrm{e}} .
\end{gathered}
$$

Taking into account (43) we deduce easily that

$$
\mathfrak{R}\left\{\mathrm{D}_{0}\left(\eta_{\mathrm{e}} \mathrm{n}_{\mathrm{o}} \xi-\xi \mathrm{D}_{0} \eta_{\mathrm{e}}\right)\right\} \square 0,
$$




$$
\begin{array}{r}
\mathfrak{R}\left(\eta_{e} D_{0} \xi+\xi D_{0} \eta_{e}\right)=0, \\
\mathfrak{R}\left\{\left(\frac{3}{2} \varkappa_{0}-N\right)\left(\eta \eta_{e}-\xi \zeta_{e}\right\}\right)=0 .
\end{array}
$$

Applying the averaging operator to both sides of (53) and using (54), we deduce the basic equation for the determination of $\cdot c$ :

$$
\mathfrak{M}\left(r_{\mathrm{c}} \mathbf{F}\right)-2 \xi \mathrm{c} \mathfrak{R}\left[r_{\mathrm{e}}\left(\mathrm{D}_{0}+1-\mathrm{m}\right) \Lambda \xi_{\mathrm{e}}\right]=0 .
$$

The computation of terms $c_{2}, c_{4}, \ldots$ in $c c$ is closely associated with the computation of terms with the characteristics of the type $e \lambda^{2}$, where, is a monomial in basic parameters. These characteristics produce the term $c, 2$ of order $:^{2}$ in the expansion of $c$. Designating by

$$
F_{e^{, 2} \cdot c}=e \cdot 2 \sum\left(x_{i} b^{+2 i+c} \cdot a_{-i}^{\prime} \zeta^{-2 i-c}\right)
$$

the aggregate of terms with the characteristic e $i^{2}$ and with the exponents $\pm(2 i+c)$, we obtain from (55)

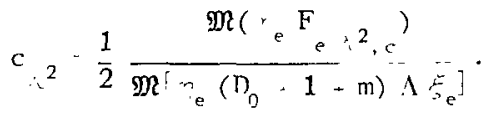

The terms whose characteristics are different from the type mentioned above do not contribute to

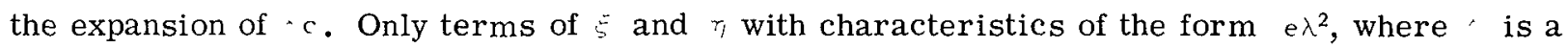
monomial in the basic parameters, contribute terms of the order of $:{ }^{2}$ to the expansion of $c$. We obtain the differential equations for the terms of higher orders and higher characteristics in the developments of $\xi$ and $\gamma$ by expanding $F$, as given by Equation (52), in powers of the basic parameters. Substituting

$$
\begin{aligned}
& \delta \mathrm{c}=\mathrm{c}_{2}+\mathrm{c}_{4}+\cdots \\
& \delta \mathrm{D}=\mathrm{D}_{2}+\mathrm{D}_{4}+\cdots \\
& \xi=\xi_{2}+\xi_{3}+\cdots \\
& \eta=\eta_{2}+\eta_{3}+\cdots \\
& \chi=x_{1}+x_{2}+\cdots
\end{aligned}
$$

and using (26), (33) - (34'), we can obtain the expansion of

$$
-\left[2\left(D_{0}+1+m\right) \delta D+(\delta D)^{2}\right] \xi-\xi_{e}(\delta C)^{2}+S
$$


in $\mathrm{F}$ without difficulty. The development of the first term

$$
-\frac{\partial \Omega}{\partial \eta}+\left(\frac{\partial \Omega}{\partial \eta}\right)_{1}
$$

requires the application of the operator

$$
\tau=\exp \left(\xi \frac{\partial}{\partial \xi_{0}}+\eta \frac{\partial}{\partial \eta_{0}}+z \frac{\partial}{\partial z_{0}}\right)
$$

in its expanded form to $-\partial \Omega / \curvearrowright \eta$, where $\xi, \eta$ and $z$ are replaced by $\xi_{0}, \eta_{0}$ and $z_{0}$, respectively. The value of $z_{0}$ is set equal to zero after the differentiation.

The expansion of $\tau$ has the same form as $\mathrm{T}$ in (31) with

$$
\begin{gathered}
\partial_{k}-\bar{\partial}_{k} \frac{\partial}{\partial \eta_{0}}+\eta_{i} \frac{\partial}{\partial \eta_{0}}+z_{k} \frac{\partial}{\partial z_{0}} \\
<1,2,3, \ldots
\end{gathered}
$$

and Equations (32) and $\left(32^{\circ}\right)$ can be used to obtain the expansions of the corresponding ${ }^{\top}{ }_{i j}$-operators in terms of

$$
\bar{n}_{1}, \check{5}_{2}, \ldots, n_{1}, r_{2}, \ldots, z_{1}, z_{2}, z_{3}, \ldots
$$

From the aggregate of terms of a given order we must extract the terms with a given characteristic. Such an extraction operator is imbedded into analytical programming languages. In FORMAC, for example, it is associated with the COEFF-symbol. A typical differential equation which we have to solve has the form

$$
\left[\left(n_{0} \cdot 1 \cdot m\right)^{2}+\frac{1}{2}\left(n_{n} \cdot m^{2}\right)\right]=\frac{3}{2} \alpha_{0} \cdot M r+N \eta+\Phi_{\lambda}
$$

where $\Phi_{\lambda}$ are the cross-effect terms with the characteristic. . We may start the iteration by neglecting $\mathrm{M}_{\xi}$ and $\mathrm{N} \eta$ and repeat the iterative process till we reach the numerical convergence. The typical differential equation we have to deal with at each iteration step has the form

$$
\left[\left(D_{0}+1-m\right)^{2}+\frac{1}{2}\left(\kappa_{0}-m^{2}\right)\right]=+\frac{3}{2} \kappa_{0} \eta \cdot \lambda \sum_{1}\left(A_{i} \zeta^{+2 i+2}+A_{-i}^{\prime} \zeta^{-2 i-\hat{z}}\right) .
$$

Its solution is

$$
\xi-\lambda \sum_{i}\left(\nu_{i} \gamma_{3}^{+2 i+2}+\nu_{-i}^{\prime} \zeta^{-2 i-j}\right),
$$

where

$$
\nu_{1}=l i, \text {, } i A_{1}+(i, a) A_{-i}^{\prime},
$$




$$
\nu_{-i}^{\prime}=(i, a) A_{i}+\{i, a\} A_{-i}^{\prime},
$$

and where we set

$$
\begin{aligned}
& {[i, a]=\frac{(2 i+a-1-m)^{2}+\frac{1}{2}\left(\kappa_{0}+m^{2}\right)}{(2 i+a)^{2}\left[(2 i+\alpha)^{2}-\kappa_{0}+2 m^{2}\right]},} \\
& (i, a)=-\frac{3}{2} \frac{\kappa_{0}}{(2 i+a)^{2}\left[(2 i+a)^{2}-\kappa_{0}+2 m^{2}\right]}, \\
& \{i, \alpha\}=\frac{(2 i+a+1+m)^{2}+\frac{1}{2}\left(\kappa_{0}+m^{2}\right)}{(2 i+a)^{2}\left[(2 i+x)^{2}-\kappa_{0}+2 m^{2}\right]} .
\end{aligned}
$$

The imposition of condition (50) has an interesting and important implication. If the characteristic has the form $e^{\lambda^{2}}$, then the right side of (56) and the corresponding parts of $\xi$ and $\eta_{y}$ contain the terms $\zeta^{+c}$ and $\zeta^{-c}$. As a consequence of (50) the coefficients $\nu_{0}$ and $\nu_{0}^{\prime}$ will be equal (Brown, 1896 and 1899)

$$
\nu_{0}^{\prime}=\nu_{0}^{\prime}
$$

If we use $(57)-(61)$ to determine $\nu_{0}$ and $\nu_{0}^{\prime}$, we shall have small numbers in the numerators and denominators, and consequent loss of accuracy. Knowledge of (62) resolves the difficulty associated with the appearance of a spurious small divisor. From (56') and (56") we obtain at each iteration step

$$
\begin{gathered}
{\left[\left(c_{0}+1-m\right)^{2}+\frac{1}{2}\left(\kappa_{0}-m^{2}\right)\right] \nu_{0}+\frac{3}{2} \kappa_{0} \nu_{0}^{\prime}=A_{0},} \\
{\left[\left(c_{0}-1-m\right)^{2}+\frac{1}{2}\left(\kappa_{0}+m^{2}\right)\right] \nu_{0}^{\prime}+\frac{3}{2} \kappa_{0} \nu_{0}=A_{0}^{\prime},}
\end{gathered}
$$

and, taking (62) into account, we deduce

$$
\begin{aligned}
& \nu_{0}=\nu_{0}^{\prime}=A_{0} /\left[\left(c_{0}+1-m\right)^{2}+2 \kappa_{0}+\frac{1}{2} m^{2}\right], \\
& \nu_{0}=\nu_{0}^{\prime}=A_{0}^{\prime} /\left[\left(c_{0}-1-m\right)^{2}+2 \kappa_{0}+\frac{1}{2} m^{2}\right] ;
\end{aligned}
$$


$\nu_{0}$ should be determined from (63). Equation (64), because of the relative smallness of the denominator, gives a less accurate result, but can be used for a check.

\section{PERTURBATIONS IN THE Z-COORDINATE}

We transform the differential equation (3) to the form suitable for computation of $z$ and $g_{0}$ by the process of iteration. We have from (3)

$$
\left(D_{0}^{2}-m^{2}-\kappa w_{0}^{3}\right) z=-\left[2 D_{0} \delta D+(\delta D)^{2}-\kappa\left(w^{3}-w_{0}^{3}\right)\right] z-\frac{1}{2} \frac{\partial \Omega}{\partial z}
$$

This form of the differential equation for $z$ emphasizes the role of the operator $2 \mathrm{D}_{0} \delta \mathrm{D}$ in formation of the linear equation for terms of the expansion of $\mathrm{g}$. The term

$$
-\left[2 D_{0} \delta D+(\delta D)^{2}-k\left(w^{3}-w_{0}^{3}\right)\right] z
$$

contains cross-effects. Using the substitution

$$
z=\gamma\left[\left(1+\zeta_{0}\right) \zeta^{+g}-\left(1+\eta_{0}\right) \zeta^{-g}\right]+\psi
$$

which resembles the substitution in the theory by Andoyer (1926), and taking (21) and (22) into account, we obtain instead of (65)

$$
\begin{aligned}
& \left(D_{0}^{2}-m^{2}-k\right) \psi=k\left(w_{0}^{3}-1\right) \psi \\
& +2 \gamma\left(1+\mathrm{m}-\mathrm{g}_{0}\right)\left(\xi^{+\mathrm{g} D} \xi_{0}+\zeta^{-\mathrm{g} D} \eta_{0}\right) \\
& +\gamma\left(\kappa_{0}+\mathrm{m}^{2}-\mathrm{g}_{0}^{2}\right)\left[\left(1+\xi_{0}\right) \zeta^{+\mathrm{g}}-\left(1+\eta_{0}\right) \zeta^{-\mathrm{g}}\right] \\
& +\frac{3}{2} \mathrm{~m}^{2} \gamma\left[\left(1+\eta_{0}\right) \zeta^{+\mathrm{g}-2}-\left(1+\xi_{0}\right) \zeta^{-\mathrm{g}+2}\right]-2 \mathrm{D}_{0} \& \mathrm{D} z_{1}+Z,
\end{aligned}
$$

where we set

$$
Z=-(\delta \mathrm{D})^{2} z_{1}-\left[2 \mathrm{D}_{0} \delta \mathrm{D}+(\delta \mathrm{D})^{2}\right]\left(z-z_{1}\right)-\kappa\left(\mathrm{w}^{3}-\mathrm{w}_{0}{ }^{3}\right) z-\frac{1}{2} \frac{\partial \Omega}{\partial z}
$$

or, taking into account (17) and

$$
\begin{gathered}
\Gamma^{2} z_{1}=z_{1}, \\
Z=-z_{1}(\delta g)^{2}-\left[2 D_{0} \delta D+(\delta D)^{2}\right]\left(z-z_{1}\right)-\kappa\left(3 w_{0}^{5} x+B\right) z-\frac{1}{2} \frac{\partial \Omega}{\partial z} .
\end{gathered}
$$


Equation (67) is simpler than the corresponding equation of Andoyer. It has the form which emphasizes the relative importance of different terms in $\psi$ and, because of the presence of the small parameter $m^{2}$ in $w_{0}^{3}-1$, it is suitable for solving by the iterative process. Setting

$$
\psi=\psi_{1}+\sigma, \quad \sigma=z_{2}+z_{3}+\cdots
$$

where ${ }_{1}$ is of the first order in $\gamma$ and $\sigma$ is the aggregate of all terms of higher order and higher characteristics. We can split equation (67) into two equations,

$$
\begin{aligned}
\left(\mathrm{D}_{0}^{2}\right. & \left.-\mathrm{m}^{2}-\kappa\right) \psi_{1}=\kappa\left(\mathrm{w}_{0}^{3}-1\right) \dot{1}_{1} \\
& +2 \gamma\left(1+\mathrm{m}-\mathrm{g}_{0}\right)\left(\zeta^{+\mathrm{g}} \mathrm{D}_{0} \xi_{0}+\zeta^{-g} \mathrm{D}_{0} \eta_{0}\right) \\
& +\gamma\left(\kappa_{0}+\mathrm{m}^{2}-\mathrm{g}_{0}^{2}\right)\left[\left(1+\xi_{0}\right) \zeta^{+\mathrm{g}}-\left(1+\eta_{0}\right) \zeta^{-\mathrm{g}}\right] \\
& +\frac{3}{2} \mathrm{~m}^{2} \gamma\left[\left(1+\eta_{0}\right) \zeta^{+\mathrm{g}-2}-\left(1+\xi_{0}\right) \zeta^{-\mathrm{g}+2}\right],
\end{aligned}
$$

and

$$
\left(\mathrm{D}_{0}^{2}-\mathrm{m}^{2}-\kappa\right) \sigma=\kappa\left(w_{0}^{3}-1\right) \mathrm{s}+2-2 \delta \mathrm{g} \mathrm{D}_{0} \Gamma z_{1}
$$

The coefficient of the typical term

$$
\zeta^{+g+2 j}-\zeta^{-2 g-2 j}, \quad j \leq-1
$$

in the expansion of the right side of the equation (68) will be of the order of $\mathrm{m}^{2 \mid j !}$. Thus these terms will decrease very rapidly. The term

$$
\zeta^{+g-2}-\zeta^{-g+2}
$$

constitutes an exception, because it will be affected by a small divisor in the process of integration. Originally of the order of $\mathrm{m}^{2}$, this term will be of the order of $\mathrm{m}$ after the integration is performed, and will be the largest term in the expansion of $\psi_{1}$. The value of $\mathrm{g}_{0}$ is obtained from the condition that no term of the form

$$
\zeta^{4} \mathrm{~g}-\zeta^{-\mathrm{g}}
$$

should be present in $\psi_{1}$. Substituting (35) and (36) into (68), and designating by $K_{0}$ the coefficient at the term

$$
\zeta^{+B}-\zeta^{-B}
$$

in the expansion of $\kappa\left(w_{0}^{3}-1\right) \psi_{1}$, we have by suppressing this term in the right side of $(68)$ : 


$$
\mathrm{g}_{0}^{2}=\kappa_{0}+\mathrm{m}^{2}+\frac{3}{2} \mathrm{~m}^{2} \mathrm{a}_{-1}+K_{0}
$$

In the last relation, only $K_{0}$ varies from iteration to iteration. This method of computing $g_{0}$ can replace a method based on the use of the determinantal equation or its analytic equivalent. Another method we suggest is the application of Andoyer's expansion to Adams' equation (1877):

$$
D_{0}^{2} z_{1}=\left(\kappa w_{0}^{3}+m^{2}\right) z_{1}=z_{1} \sum_{-\infty}^{+\infty} S_{2 j} \zeta^{2 j}
$$

Substituting the expansions of $\xi_{0}$ and $\eta_{0}$ into (68) and keeping only the most influential term, we obtain an approximate differential equation for $\psi_{1}$ :

$$
\begin{gathered}
{\left[D_{0}^{2}-m^{2}-\kappa\left(1-\frac{3}{2} a_{+1}^{2}-\frac{3}{2} a_{-1}^{2}\right)\right] \psi_{1}} \\
=\gamma\left\{\left[\left(1-2 m+\frac{5}{2} m^{2}\right)-\left(g_{0}-2\right)^{2}\right] \quad a_{-1}+\frac{3}{2} m^{2}\right\}\left(\zeta^{+g-2}-\zeta^{-k+2}\right) .
\end{gathered}
$$

The solution of this differential equation

$$
\psi_{1}=\frac{\left[\left(1-2 m+\frac{5}{2} m^{2}\right)-\left(g_{0}-2\right)^{2}\right] a_{-1}+\frac{3}{2} m^{2}}{\left(g_{0}-2\right)^{2}-m^{2}-\kappa\left(1-\frac{3}{2} a_{+1}^{2}-\frac{3}{2} a_{-1}^{2}\right)}\left(y_{2}^{+g-2}-\zeta^{-k+2}\right)
$$

serves to start the iterative process. At each iteration step, equation (68) has the form

$$
\begin{gathered}
\left(D_{0}^{2}-m^{2}-\kappa\right) \psi_{1}=\sum_{j} M_{1}\left(\zeta^{+}+2^{+}-\zeta^{-g-2 j}\right) \\
j=1,2,3, \ldots, M_{j}=0\left(m^{2 j}\right)
\end{gathered}
$$

and the solution is

$$
\dot{\psi}_{1}=\sum_{j} k_{j}\left(\zeta^{+g+2 j}-\zeta^{-g-2 j}\right)
$$


where

$$
k_{j}=M_{j} /\left[\left(g_{0}+2 j\right)^{2}-m^{2}-\kappa\right]
$$

If $\mathrm{g}_{0}$ is known in advance, then the only term in the right-hand side of (68) which changes from iteration to iteration is

$$
r\left(w_{0}^{3}-1\right) \psi_{1}
$$

The other terms, and the denominator in (72), remain the same from iteration to iteration.

Multiplying equation (69) by $z_{1}$ and equation

$$
\left(\mathrm{D}_{0}^{2}-\mathrm{m}^{2}-r \mathrm{w}_{0}^{3}\right) z_{1}=0
$$

by s, and subtracting the results, we obtain

$$
D_{0}\left(z_{1} D_{0} \sigma-\sigma D_{0} z_{1}\right) z_{1} Z-2 z_{1} \partial g D_{0} I^{\prime} z_{1}
$$

Taking into account

$$
\left.\mathfrak{R}+\mathrm{D}_{0}\left(\mathrm{z}_{1} \mathrm{D}_{0} \sigma-\sigma \mathrm{D}_{0} \mathrm{z}_{1}\right)\right\}=0,
$$

we deduce from Equation (73)

$$
\mathfrak{M}\left(z_{1} Z\right)-2 ; q \mathfrak{M}\left(z_{1} D_{n}: z_{1}\right)-0
$$

or

$$
\begin{gathered}
\left.\mathfrak{R},\left(1-\gamma_{n}\right)+\mathfrak{k}-\left(1+\gamma_{0}\right) \zeta^{-g}+\psi_{1}^{j} z\right\rangle \\
-2-g \mathfrak{M}\left(z_{1} D_{0} \Gamma z_{1}\right)-0 .
\end{gathered}
$$

Equation (74) or (75) serves for the determination of,$g$. Evidently only these parts of $F$ having characteristics of the form $;,^{2}$, where, is a monomial in $e, e^{\prime}, \gamma$ and $x$, contribute to the expansion of $g$. They produce terms of the order of $\times 2$ in $\delta g$.

We derive the differential equations for the terms of higher orders and higher characteristics in $\sigma$ by expanding $Z$ in powers of the basic parameters.

Substituting

$$
\begin{aligned}
& \therefore g-\mathrm{g}_{2}+\mathrm{g}_{4}+\cdots \\
& \therefore \mathrm{D} \quad \mathrm{D}_{2}+\mathrm{D}_{4}+\cdots \\
& x \quad \lambda_{1}-x_{2}+\cdots
\end{aligned}
$$




$$
\begin{aligned}
& \sigma=z_{2}+z_{3}+\cdots \\
& Z=z_{1}+z_{2}+z_{3}+\cdots
\end{aligned}
$$

into (67') and taking (34) and (34') into account, we obtain the development of the first three terms in (67") without any great difficulty. The development of the fourth term is derived by applying the operator $\mathrm{T}$, in its expanded form, to $-1 / 2 \partial \Omega / \hat{\mathrm{z}} \mathrm{z}$ in which $\xi, \eta$ and $\mathrm{z}$ are replaced by $\xi_{0}$, $\eta_{0}$ and $z_{0}=0$, respectively. 


\section{CONCLUSION}

We discussed certain features which, it seems to us, are peculiar to a form of the theory of satellites in rectangular coordinates, if an analytic programming language is used.

The works of Hill and Brown can hardly be surpassed for mathematical elegance and for deep insight into the form of the expansion of coordinates and frequencies and their dependence upon the constants of integration.

Here Hill and Brown have said the last word, providing trigonometrical series are used as the means of solution. Space is left, however, for the decision as to how to derive the solution and what the most convenient form of the differential equations would be if the analytic manipulations are performed automatically.

In our approach to these problems, we found inspiration in the work of Andoyer on the Hill-Brown lunar theory. We found that it contains several interesting points, which sound quite modern. This beautiful work deserves revived attention.

We obtained Hill's periodic intermediate orbit by solving simultaneously the differential equation for the complex coordinate and the Andoyer differential equation for the parallax, bypassing the formation of the infinite system equations for coefficients of the variation terms.

We transformed the differential equations of the Hill-Brown theory to a form which contains a small parameter in the right-hand sides and thus favors the application of the method of iteration and the use of analytical programming language.

Together with the expansion of the coordinates, we derive the expansion of the parallax. Its knowledge facilitates the expansion of the right-hand sides of the differential equations in powers of the constants of integration. The right hand sides of the differential equations contain $T_{i}, j$, the polynomials in $x_{1}, x_{2} \ldots$. The representation of $T_{i}$, in the form of the polynomials in the constants of integration constitutes an essential part of the theory and programming. The knowledge of this representation facilitates greatly the formation of the differential equations for the terms with the prescribed characteristic.

We expanded the differential operators into series in squares of the constants of integration.

Only the terms of order zero in the expansions of the differential operators are employed in the integration of the differential equations. The remaining terms are responsible for producing the cross effects between the perturbations of different orders.

All programming was done by $\mathrm{R}$. Baxter, to whom the author is greatly indebted for his generous help and friendly cooperation. He is using FORMAC as a basis.

We are aware that FORMAC is not the most efficient language as far as computer time is concerned. However, it has been valuable to us in terms of flexibility and ease of programming of the numerous sub-problems into which lunar theory in coordinates is split. It was also very useful in checking the correctness and the applicability of the theoretical thought. 
We are applying the theory presented here to the seventh satellite of Jupiter and compare the results with the work of Tokmalaieva (1956).

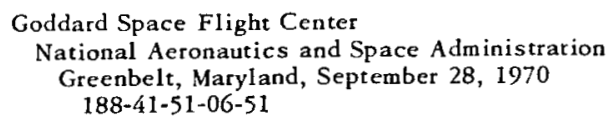

\section{REFERENCES}

1. Adams, J. C., "On the Motion of the Moon's Node," $M$. N. 38, p. 43, 1877.

2. Andoyer, H., "Cours de Mécanique Celeste," Vol. 2, 134-242, 1926.

3. Barton, D., "Lunar Disturbing Function," Astron. J. 71(6):438-422, August 1966.

4. Broucke, R., "Computerized Series Expansions: Applications to Lunar Theory," JPL Space Programs Summary 37-55, Vol. III, pp. 9-12, 1968.

5. Broucke, R. and Garthwaite, K., "A Programming System for Analytical Series Expansion on a Computer," Celestial Mechanics 1(2):271-284, 1969.

6. Brown, E. W., "Theory of the Moon," Mem. Roy. Astr. Soc. 53, pp. 39-116, 163-202, 1896-1899.

7. Charpont, J. and Mangeney, L., "Développements Littéraux dans la Théorie Analytique de la Lune," Astron, and Astroph. 2(4):425-445, August 1969.

8. Danby, J. M. A., Deprit A., and Rom, A. R. M., "The Symbolic Manipulation of Poisson Series," BSRL Note 432, Boeing Scientific Research Laboratories, 1965.

9. Deprit, A., "Canonical Transformations Depending on a Small Parameter," Celestial Mechanics (1):12-30, January 1969.

10. Deprit, A, and Henrard, J., "Analytical Lunar Ephemeris: Delaunay's 'Theory Revisited," Science 168(3939):1569-1570, June 26, 1970.

11. Elmabsout, B., "Méthode Semi-numérique de Résolution du Problem de Hill-Application à Phoebe," Astron. and Astrophys. 5:68-83, 1970.

12. Faa de Brouno, "Note sur une Nouvelle Formule de Calcul Differential, Quarterly Journ. Math. 1, p. $359,1855$.

13. Hill, G. W., "Researches in the Lunar Theory, Am. Journ. Math. I, pp. 5-26, 129-147, 245-260, 1878. "On the Part of the Motion of the Lunar Perigee Which is a Function of the Motion of the Sun and the Moon," Acta Math. 8, pp 1-36, 1886.

14. Kamel, A. A., "Expansion Formulae in Canonical Transformations Depending on a Small Parameter," Celestial Mechanics, 1(2):190-199, 1969.

15. Tokmalaieva, S. S., "Analytical Theory of Motion of the Seventh Satellite of Jupiter (In Russian). Trudy I. T. A. Leningrad, 1956. 


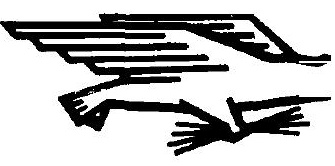

POSTAGE AND FEES- PA NATIONAL AERONAUTICS SPACE ADMINISTRATIO

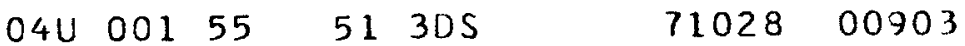

AIR FORCE WEAPONS LABORATORY /WLOL/

KIRTLAND AFB, NEW MEXICO 87117

ATT E. LOU BOWMAN, CHIEF, TECH. LIBRARY

POSTMASTER:

If Undeliverable (Section Postal Manual) Do Not R

"The aeroniutical and space activities of the United States shall be conducted so as to contribute . . to the expansion of buman knowledge of phenomena in the atmosphere and space. The Administration shall provide for the videst practicalle and appropriate dissemination of information concerning its activities and the results thereof."

- National Aeronautics ANd SPACE ACt of 1958

\section{NASA SCIENTIFIC AND TECHNICAL PUBLICATIONS}

TECHNICAL REPORTS: Scientific and technical information considered important, complete, and a lasting contribution to existing knowledge.

TECHNICAL NOTES: Information less broad in scope but nevertheless of importance as a contribution to existing knowledge.

TECHNICAL MEMORANDUMS: Information receiving limited distribution because of preliminary data, security classification, or other reasons.

CONTRACTOR REPORTS: Scientific and technical information gènerated under a NASA contract or grant and considered an important contribution to existing knowledge.
TECHNICAL TRANSLATIONS: Information published in a foreign language considered to merit NASA distribution in English.

SPECIAL PUBLICATIONS: Information derived from or of value to NASA activities. Publications include conference proceedings, monographs, data compilations, handbooks, sourcebooks, and special bibliographies.

TECHNOLOGY UTILIZATION PUBLICATIONS: Information on technology used by NASA that may be of particular interest in commercial and other non-aerospace applications. Publications include Tech Briefs, Technology Utilization Reports and

Technology Surveys.

Details on the availability of these publications may be obtained from:

SCIENTIFIC AND TECHINICAL INFORMATION OFFICE

NATIONAL AERONAUTICS AND SPACE ADMINISTRATION

Washington, D.C. 20546 